\title{
BMJ Open Ross for Valve replacement In AduLts (REVIVAL) pilot trial: rationale and design of a randomised controlled trial
}

\author{
Richard Whitlock (10 , ${ }^{1}$ Emilie Belley-Cote, ${ }^{2}$ Filip Rega, ${ }^{3}$ Michael W.A. Chu, ${ }^{4}$ \\ Graham R McClure, ${ }^{1}$ Hanna Hronyecz, ${ }^{5}$ Peter Verbrugghe, ${ }^{3}$ PJ Devereaux, ${ }^{2}$ \\ Shrikant Bangdiwala, ${ }^{6}$ John Eikelboom, ${ }^{2}$ Katheryn Brady, ${ }^{6}$ Ravil Sharifulin, ${ }^{7}$ \\ Alexander Bogachev-Prokophiev, ${ }^{7}$ Serban Stoica ${ }^{8}$
}

To cite: Whitlock R, BelleyCote E, Rega F, et al. Ross for Valve replacement In AduLts (REVIVAL) pilot trial: rationale and design of a randomised controlled trial. BMJ Open 2021;11:e046198. doi:10.1136/ bmjopen-2020-046198

- Prepublication history for this paper is available online To view these files, please visit the journal online (http://dx.doi. org/10.1136/bmjopen-2020046198).

Received 24 October 2020 Accepted 20 June 2021

Check for updates

(c) Author(s) (or their employer(s)) 2021. Re-use permitted under CC BY-NC. No commercial re-use. See rights and permissions. Published by BMJ.

For numbered affiliations see end of article.

Correspondence to Dr Richard Whitlock Richard.Whitlock@phri.ca

\section{ABSTRACT}

Introduction In non-elderly adults, aortic valve replacement (AVR) with conventional prostheses yield poor long-term outcomes. Recent publications suggest a benefit of the Ross procedure over conventional AVR and highlight the need for high-quality randomised controlled trial (RCTs) on the optimal AVR. We have initiated a pilot trial assess two feasibility criteria and one assumption: (1) evaluate the capacity to enrol six patients per centre per year in at least five international centre, (2) validate greater than $90 \%$ compliance with allocation and (3) to validate the proportion of mechanical $(\geq 65 \%)$ vs biological $(\leq 35 \%)$ valves in the conventional arm.

Methods and analysis Ross for Valve replacement In AduLts (REVIVAL) is a multinational, expertise-based RCT in adults aged 18-60 years undergoing AVR, comparing the Ross procedure versus one of the alternative approaches (mechanical vs stented or stentless bioprosthesis). The feasibility objectives will be assessed after randomising 60 patients; we will then make a decision regarding whether to expand the trial with the current protocol. We will ultimately examine the impact of the Ross procedure as compared with conventional AVR in non-elderly adults on survival free of valve-related life-threatening complications (major bleeding, systemic thromboembolism, valve thrombosis and valve reoperation) over the duration of follow-up. The objectives of the pilot trial will be analysed using descriptive statistics. In the full trial, the intentionto-treat principle will guide all primary analyses. A timeto-event analysis will be performed and Kaplan-Meier survival curves with comparison between groups using a log rank test will be presented.

Ethics and dissemination REVIVAL will answer whether non-elderly adults benefit from the Ross procedure over conventional valve replacement. The final results at major meetings, journals, regional seminars, hospital rounds and via the Reducing Global Perioperative Risk Multimedia Resource Centre.

Trial registration number ClinicalTrials.gov Identifier: NCT03798782

Protocol version January 29, 2019 (Final Version 1.0)

\section{INTRODUCTION}

Approximately 85000 patients undergo aortic valve replacement (AVR) annually in the USA
Strengths and limitations of this study

- A large randomised controlled trial of the Ross procedure versus conventional valve replacement powered for clinical outcomes.

- Patient engagement in trial design.

- Expertise-based randomisation.

- Long follow-up for a disease state that is lifelong.

Blinding of physicians and patients is not feasible.

alone. ${ }^{1}$ AVR options include a mechanical valve, biological valve or the Ross procedure. The conventional procedure is to use either a mechanical or biological valve. Although conventional AVR improves survival and quality of life in patients with severe aortic valve disease, ${ }^{2}{ }^{3}$ long-term survival after surgery remains lower than the general population. ${ }^{2}$ The expected lifespan after AVR using mechanical or biological valves in non-elderly adults is approximately halved compared with matched patients who do not require AVR. ${ }^{4}$ Mechanical valves confer the majority of their risk and negative effect on quality of life through prosthesis thrombogenicity, which necessitates lifelong anticoagulation with its bleeding risk. Biological valves can reduce this risk, but at the cost of limited prosthesis durability. A 35-year-old patient receiving a bioprosthetic valve has a reoperation-free life expectancy of 10 years and a $63 \%$ lifetime risk of reoperation. ${ }^{4}$ The Ross procedure replaces a patient's diseased aortic valve with their own pulmonic valve (pulmonary autograft), while a pulmonary homograft is implanted in the pulmonary position. ${ }^{56}$ Proponents of the procedure suggest the operation is superior to conventional valve replacement because the autograft is a living, dynamic structure providing superior haemodynamics, a lower risk of thromboembolism (with no 
long-term anticoagulation requirement), and a lower risk of endocarditis. ${ }^{7-10}$

To date, single-centre reports and registry data regarding the Ross procedure have not impacted guideline recommendations regarding AVR choice in non-elderly adults. The existing evidence suggesting a lower incidence of thromboembolism, bleeding or valve-related events with the Ross procedure compared with conventional valve replacement is graded as low quality. ${ }^{11}$ Furthermore, as the pulmonary homograft is implanted into the lowpressure pulmonary circulation, it is proposed to be less vulnerable to structural degeneration than homografts or bioprostheses exposed to systemic pressures. ${ }^{7-10}$ Critics argue that the complexity of the Ross procedure increases operative risk and converts a single valve disease into a two-valve disease with the potential for homograft dysfunction leading to right ventricular outflow tract obstruction, requiring either surgical or percutaneous reintervention. $^{12} 13$ These concerns have led to uncertainty regarding the actual risks and benefits of the Ross procedure compared with conventional AVR in nonelderly patients. ${ }^{415}$

A large retrospective cohort from UK National Registry Data described 1501 congenital heart patients who underwent AVR, of which $47.8 \%$ had the Ross procedure, $37.8 \%$ had a mechanical prosthesis, $10.9 \%$ had a bioprosthesis and $3.5 \%$ had a homograft. After matching these patient groups by propensity and restriction and, using a Bayesian dynamic survival model, the Ross procedure was superior to mechanical and bioprosthetic valves for survival and freedom from reoperation. ${ }^{16}$ The Ross procedure produced similar survival to the matched general population who did not require AVR, while no other AVR technique achieved this outcome.

Mazine et al reported an observational study with 20-year follow-up that matched 208 Ross patients with patients who received a mechanical valve (mean age 34 years). They demonstrated that long-term survival and freedom from reintervention were comparable between the Ross procedure and mechanical AVR. ${ }^{7}$ However, the Ross procedure was associated with improved freedom from cardiac and valve-related mortality, as well as a significant reduction in the incidence of stroke or major bleeding. The largest randomised controlled trial (RCT) assessing the Ross procedure versus aortic homograft was a single-centre, single-surgeon trial $(\mathrm{n}=216)$. This trial demonstrated that patients who had a homograft were at increased mortality risk compared with patients who had a Ross procedure. Furthermore, in that trial, the Ross procedure had survival similar to that of an age and sexmatched general population. The generalisability of this single centre trial is unknown.

We completed two systematic reviews on the Ross procedure: the first compared Ross to conventional AVR on survival, reintervention and adverse valve-related events and the second refined event rate estimates by pooling 66 studies non-comparative Ross studies and performed a microsimulation modelling to evaluate age-specific and sex-specific life expectancy for patients undergoing the Ross procedure. ${ }^{11} 17$ In the comparative meta-analysis, fifteen studies (2 RCTs, 13 observational) evaluating the Ross procedure vs conventional AVR in adult patients were included for final analysis. Our results suggest that the Ross procedure does not increase mortality within 30 days, but significantly reduces the risk of mortality on longer-term follow-up. In addition, the risk for reoperation to either the pulmonic or aortic valve was not significantly different, while risks of ischaemic stroke and major bleeding were significantly lower with the Ross procedure. Based on the GRADE framework, ${ }^{18}$ these data are of very low quality, resulting in very low confidence in the point estimates of effect.

Given the unacceptably poor outcomes among nonelderly patients requiring conventional AVR and the encouraging Ross procedure data, a multicentre, expertise-based RCT powered to assess long-term clinically relevant outcomes comparing the Ross procedure with conventional AVR is needed. We describe the methods of the a multicentre pilot, but also briefly describe the current concept of the full trial. Aspects of the full trial may change based on experience gained in the pilot phase.

\section{Trial design}

Pilot trial

The Ross for Valve replacement In AduLts (REVIVAL) pilot trial is a 3-year, 60-patient, expertise-based RCT ${ }^{19}$ that will be conducted in approximately five international centres. It will assess whether it is feasible to enrol 1047 patients into a large RCT in over 50 centres within 3 years, evaluating the efficacy and safety of the Ross procedure. The pilot participants will be followed for approximately 3 years. If the pilot demonstrates the feasibility of the full trial, without assessing the outcomes for the main trial we will include these patients in the full trial and their follow-up will be extended for a median of 7 years.

\section{Full trial}

The REVIVAL full trial is a multicentre, expertise-based, RCT evaluating the efficacy and safety of the Ross procedure compared with conventional AVR in non-elderly patients with aortic valve disease. The target recruitment is 1047 patients from 50 international centres. The primary hypothesis is that the Ross procedure will improve survival free of life-threatening valve complications, conferring significant benefit over conventional AVR.

Patients are stratified based on conventional AVR (ie, mechanical vs bioprosthetic) treatment choice before randomisation. Additionally, randomisation is stratify by centre to account for differences in local patient populations and cointerventions. Eligible and consenting patients are randomised via the central interactive web randomisation system at the Population Health Research Institute (PHRI), Canada. As per the expertise-based design, patients are randomised to receive surgery from a surgeon with expertise in the Ross procedure 
and committed to performing the Ross procedure or to surgeon with expertise in conventional AVR who is committed to performing conventional AVR, in randomly permuted blocks of 2, 4 or 6 .

Allocation is communicated to site personnel and patients at the time of randomisation to ensure adequate time is allowed for consultation with the surgeon responsible for their valve replacement. Blinding of patients or clinicians is not feasible due to logical constraints, and because of the requirement for oral anticoagulants with mechanical valves. Event adjudicators will be blinded to the best of our ability by redatcting the necessary information from source documents being used. The allocation sequence is concealed from the investigators before randomisation.

All patients are followed from the time of randomisation until the final follow-up visit. Following randomisation and baseline data collection, visits occur at hospital discharge, 30 days, 6 months and every 6 months until the common study end date (approximately 10 years after the first patient randomised). All data will be entered by the site into the central web based database at the PHRI. Data are anonymised and will be held on a secure server at the PHRI for 25 years.

\section{Selection of surgeons}

The REVIVAL trial faces issues similar to our previously published trial of off-pump versus on-pump CORONARY bypass trial. Surgical trials face some specific methodological challenges. Because of training and experience, individual surgeons are generally more proficient in a particular technique, and so are likely to primarily use a single surgical approach. ${ }^{20}$ This could compromise the validity of a conventional RCT as surgical expertise may be skewed toward the technique which is best established, most widely used or easiest to perform. ${ }^{21}$ Surgical procedures that require a learning curve are disadvantaged as a minimum number of cases need to be performed and years of experience are needed before a surgeon feels at ease with both techniques. Unless similar numbers of participating surgeons have expertise in both procedures, differential cross-over in the two arms of the trial may occur. For this reason, as we did in the CORONARY trial, we are using surgical expertise-based randomisation. ${ }^{22}$ Patients are randomised at each site a surgeon with expertise in the Ross procedure and committed to performing the Ross procedure or a surgeon with expertise in conventional AVR who is committed to performing AVR.

Surgeons are allowed to participate in the trial and perform only the procedure in which they have expertise, minimising the learning curve and decreasing the risk of crossover. We have defined 'expertise in the Ross procedure' as experienced cardiac surgeons with $\geq 2$ years of independent experience, who have performed $\geq 40$ aortic root procedures, $\geq 30$ Ross procedures with at least 8 procedures per year and $\geq 15$ aortic root procedures per year on average in their current practice. Aortic root procedures are defined as procedures requiring coronary artery reimplantation. Surgeons are considered to have 'expertise in conventional AVR' if they have at least 2 years of experience operating independently and have performed $\geq 50 \mathrm{AVR}$. Surgeons will be allowed to perform all operations for which they meet expertise criteria within this trial. Conversions from the assigned technique (crossover and reason) are recorded. Sites will begin recruitment only when all country regulatory requirements are met, the site has received research ethics board approval, all required site documents have been submitted to the central team, and the site has completed the site initiation visit.

\section{Patient selection}

Patients undergoing clinically indicated AVR are eligible if they: (1) are age 18-60 years and (2) provided written informed consent.

Patients are excluded if they (1) are undergoing concomitant coronary artery bypass grafting or another valve procedure during AVR; have (2) a known connective tissue disease; (3) severe (grade 3 or 4) right or left ventricular dysfunction; (4) pulmonic valve dysfunction or anomaly incompatible with the Ross procedure; (5) a life expectancy less than 5 years or (6) documented severe aortic insufficiency not solely due to leaflet issue. Furthermore, patients who have had a previous valve replacement not in the aortic position or previous intervention on the pulmonic valve are excluded.

We expect these criteria to exclude a very small proportion of patients given the low incidence of concomitant cardiac pathology in young adult patients. Patients will be consented by the local investigator or their research team delegates.

\section{Intervention}

The intervention under investigation is the Ross procedure compared with conventional AVR. Consenting participants will be randomised to receive definitive surgical intervention for the management of aortic valve disease by either the Ross procedure (Ross arm) using standard local technique or by conventional AVR (conventional AVR arm) defined as including three treatment modalities; stented or stentless bioprosthesis, or mechanical prosthesis implantation. All types of prostheses are allowed in this trial.

Postoperatively, physicians will manage patients as they see appropriate. Centres are encouraged to maintain their established standard of perioperative care for each group. Finally, should transcatheter aortic valves develop indication for use in this lower risk, younger population during the REVIVAL trial-these patients will be permitted into the trial in the conventional arm and count as bioprostheses. All relevant risks and benefits are explained in full to allow an informed patient and surgeon joint decision, which mirrors standard practice.

\section{Study outcomes}

Pilot trial

The pilot trial will assess several key parameters for the feasibility of the full trial. The outcome measures of the 
pilot trial are: to evaluate the capacity to enrol a mean of six patients per centre per year; to validate the assumption of greater than $90 \%$ compliance with allocation; and to validate the proportion of mechanical (at least $65 \%$ ) vs biological (at most 35\%) valves in the conventional arm which is important for estimates of event rates in the conventional group.

\section{Full trial}

The primary outcome is survival free of life-threatening valve-related complications, which are non-perioperative major bleeding, stroke or systemic thromboembolism, valve thrombosis and operated-on valve reintervention over duration of follow-up. The primary outcome was established based on a survey of 31 Ross experts, and validated in a patient engagement programme.

The secondary outcomes over the duration of follow-up are: (1) the individual components of the primary outcome (non-perioperative major bleeding, stroke or systemic thromboembolism, valve thrombosis and operated-on valve reintervention); (2) mortality within 30 days postoperatively, (3) perioperative major bleeding, (4) health-related quality of life (assessed using the SF-36 questionnaire), (5) operated-valve endocarditis, (6I) aortic valve reintervention, (7) pulmonic valve reintervention, (8) new requirement for permanent pacemaker, (9) echocardiographic parameters, (10) pregnancy free of valve-related complications and (11) live births.

Table 1 Definition and expected annual incidence of primary outcome components for the Ross procedure and conventional AVR options

\begin{tabular}{|c|c|c|c|}
\hline \multirow[b]{2}{*}{ Outcome and definition } & \multicolumn{3}{|c|}{ Annual expected incidence } \\
\hline & $\begin{array}{l}\text { Ross } \\
\text { procedure }\end{array}$ & $\begin{array}{l}\text { Mechanical } \\
\text { valve }\end{array}$ & $\begin{array}{l}\text { Bioprosthetic } \\
\text { valve }\end{array}$ \\
\hline \multicolumn{4}{|l|}{ Mortality rate after 30 days following surgery } \\
\hline $\begin{array}{l}\text { All-cause mortality, selected rather than cardiovascular mortality, as cause-specific } \\
\text { mortality is often difficult to ascertain or define in complex cardiovascular patients in } \\
\text { whom multiend-organ dysfunction may accompany cardiovascular decline. }\end{array}$ & $0.46 \%{ }^{17}$ & $1.81 \%^{30}$ & $2.94 \%{ }^{30}$ \\
\hline \multicolumn{4}{|l|}{ Non-perioperative major bleeding } \\
\hline $\begin{array}{l}\text { Non-perioperative major bleeding occurs more than } 48 \text { hours postoperatively and is } \\
\text { defined by the International Society of Thrombosis and Hemostasis as follows: } \\
\text { 1. Fatal bleeding. } \\
\text { 2. Symptomatic bleeding in a critical area or organ, such as intracranial, intraspinal, } \\
\text { intraocular, retroperitoneal, intra-articular or pericardial, or intramuscular with } \\
\text { compartment syndrome. } \\
\text { 3. Bleeding causing a fall in haemoglobin level of } 20 \mathrm{~g} / \mathrm{L})(1.24 \mathrm{mmol} / \mathrm{L}) \text { or more, or } \\
\text { leading to transfusion of two or more units of whole blood or red cells }\end{array}$ & $0.08 \%{ }^{17}$ & $2.34 \%^{30}$ & $1.55 \%^{30}$ \\
\hline
\end{tabular}

\section{Stroke}

Stroke is acute focal brain dysfunction due to a vascular cause lasting $\geq 24$ hours in the absence of brain imaging or requires evidence of acute stroke on brain imaging $0.14 \%^{17} \quad 0.97 \%{ }^{30} \quad 0.61 \%{ }^{30}$ (ie, if there is an acute/subacute stroke documented by CT or MRI or at autopsy, the duration of symptoms/signs may be less than 24 hours). Stroke is divided into three types: ischaemic stroke, haemorrhagic stroke (ie, primary intracerebral or primary subarachnoid haemorrhage) and undetermined stroke (no neuroimaging or autopsy). Stroke does not include diffuse brain ischaemia from hypoxic-ischaemic encephalopathy. If death occurs within 24 hours, the neurological deficit must persist up to the time of death.

\section{Systemic thromboembolism}

Judged to occur where there is a clinical history consistent with an acute loss of blood $\quad 0.04 \%^{17} \quad 0.90 \%{ }^{26} \quad 0.53 \%{ }^{31}$ flow to a peripheral artery (or arteries), which is supported by objective evidence of embolism.

\section{Valve thrombosis}

Defined as any thrombus not caused by infection attached to or near an operated valve $\quad 0.04 \%^{17} \quad 0.14 \%^{26} \quad 0.07 \%{ }^{31}$ that occludes part of the blood flow path, interferes with valve function, or is large enough to warrant treatment. Valve thrombus found at autopsy in a patient whose cause of death was not valve related or found at operation for and unrelated indication is to be counted as valve thrombosis.

\section{Valve reintervention}

Any surgical or percutaneous procedure that repairs, or otherwise alters or adjusts, or $\quad 1.14 \%{ }^{17} \quad 0.57 \%^{30} \quad 1.57 \%{ }^{30}$ replaces a previously implanted prosthesis or valve.

AVR, aortic valve replacement. 
Table 1 presents the definitions and expected incidence of the primary study outcomes for the Ross procedure, mechanical AVR and bioprosthetic AVR.

\section{Outcome analysis}

Pilot trial

The feasibility objectives of the pilot trial will be analysed using descriptive statistics and $95 \%$ CIs.

\section{Full trial}

The intention-to-treat principle, in which all participants will be included in their assigned treatment groups regardless of actual surgical procedure performed, will guide all primary analyses. A time-to-event analysis will be performed and Kaplan-Meier survival curves with comparison between groups using a log rank test will be presented. Treatment effect will be estimated using an HR and 95\% CI derived by the Cox proportional hazards model. This analysis will also be performed for all components of the primary outcome. We will perform a landmark analysis of the primary outcomes occurring after 30 days.

We will evaluate secondary outcomes using the Pearson $\chi^{2}$, independent sample t-test and Wilcoxon rank sum test as deemed appropriate based on the expected distribution of the data. We will perform time-to-event analyses with Kaplan-Meier curves and logrank tests for all components of the primary outcome. Further, we will perform a recurrent events analysis of the primary outcome as secondary analysis. We will adjust secondary outcome analyses for stratifying variables. After the completion of 30-day follow-up, we will assess safety outcomes (perioperative major morbidity and mortality) using these same methods.

\section{Planned subgroup analyses}

Additional Cox models will be used to evaluate subgroups of interest: type of conventional valve-bioprosthesis versus mechanical valve, aortic annular diameter greater than $28 \mathrm{~mm}$ vs less than or equal to $28 \mathrm{~mm}$ and sex. We will use additional adjusted Cox models to evaluate for subgroup differences and interaction between subgroup factors and treatment effect.

\section{Sensitivity analysis}

We will assess robustness of results for the primary outcome using a per-protocol model and a as-treated model with time-to-event analysis. Additionally, we will conduct a sensitivity analysis to assess the effect of missing data using plausible worst-case scenario anal$\mathrm{ysis}^{23}$ as it is unlikely that patients will be lost to follow-up at random.

\section{Sample size and statistical analysis}

\section{Pilot trial}

A convenience sample size of 60 patients will be used to assess feasibility for the full trial.
Table 2 Total Sample size estimates for the full trial -7 years follow-up, $90 \%$ power, alpha $5 \%$ *

\begin{tabular}{lccccr}
\hline Proportion of & \multicolumn{5}{c}{ Proportion of Ross arm with event } \\
\cline { 2 - 6 } $\begin{array}{l}\text { conventional } \\
\text { arm with event }\end{array}$ & $\mathbf{7 \%}$ & $\mathbf{8 \%}$ & $\mathbf{8 . 7 5 \%}$ & $\mathbf{9 \%}$ & $\mathbf{1 0 \%}$ \\
\hline $20 \%$ & 466 & 567 & 661 & 697 & 870 \\
\hline $19 \%$ & 531 & 656 & 775 & 821 & 1047 \\
$18 \%$ & 613 & 771 & 925 & 986 & 1289 \\
\hline $17.36 \%$ & 677 & 862 & 1047 & 1121 & 1495 \\
$17 \%$ & 718 & 922 & 1128 & 1211 & 1635 \\
\hline $16 \%$ & 857 & 1130 & 1415 & 1532 & 2158 \\
\hline
\end{tabular}

${ }^{*}$ Assuming, 3-year recruitment with median 7 years total followup, $5 \%$ cross-over in the Ross arm and $2.5 \%$ cross-over in the conventional arm, lost to follow-up of $2 \%$.

\section{Full trial}

A total sample size of 1047 patients will allow detection of a $49.6 \%$ relative risk reduction of the primary outcome with $90 \%$ power assuming a control event rate of $19.8 \%$ at 7-year follow-up. This estimate of relative risk reduction is derived from effect estimates from the published observational data.

The sample size estimates for the primary outcome (the time to event composite outcome) are based on a logrank test for comparing the two groups. ${ }^{24}$ Table 2 presents total sample size by conventional and Ross arm event rates. We assume a median follow-up of 7 years with an accrual time of 3 years and hence total follow-up time of 10 years. We further assume a crossover of $5 \%$ in the Ross arm and $2.5 \%$ in the conventional arm, a lost to follow-up of $2 \%$, and alpha $=0.05$.

\section{Independent data safety monitoring board}

An external and independent data safety monitoring board (DSMB) will ensure patient safety, receive and review interim analyses of efficacy data, provide feedback to the steering committee, and ensure the study follows the highest standards of ethics. The DSMB chair can convene a full committee meeting at any time.

\section{Pilot trial}

The DSMB will review safety data in the pilot trial at two times points. First, when 30 patients have completed 30 days of follow-up, and then on completing the 30-day follow-up for all 60 pilot patients. The DSMB will make recommendations to stop the pilot study if it is unsafe to proceed into the full large-scale trial. Given the small sample size, no formal stopping rule is set. We rely on the judgement of an experienced DSMB, who will consider the totality of the current evidence in the context of the pilot trial's safety results.

\section{Full trial}

Two formal interim analyses will be undertaken when $50 \%$ and $75 \%$ of the expected outcomes have occurred. For efficacy, a modified Haybittle-Peto approach will be used, with reductions in events of $\geq 4 \mathrm{SD}$ in the first interim 
analysis and $\geq 3 \mathrm{SD}$ in the second will be used. Predefined boundaries will have to be exceeded in two consecutive analyses performed $\geq 3$ months apart. Given the extremeness of the monitoring boundaries and the paucity of interim analyses, we will not adjust the final $\mathrm{p}$ value at the trial end. The DSMB in making a recommendation for early stopping will also consider the consistency of the secondary endpoints and any relevant external data. For safety, increases in the rates of the primary outcome of $\geq 3 \mathrm{SD}$ (first interim analysis) and $\geq 2 \mathrm{SD}$ (second interim analysis) will be used as triggers for discussion of early stopping and reporting. The DSMB will make their recommendations to the Steering Committee after considering all available data and any relevant external data.

\section{Ethics, dissemination and discussion}

In non-elderly adults, the current standard practice for managing aortic valve disease-mechanical AVR-improves symptoms and survival. In fact, the American Heart Association (AHA) website indicates that most manufactured mechanical valves "will last throughout the remainder of the patients' lifetime.. ${ }^{25}$ What the AHA fails to mention, however, is that the life expectancy of younger patients with aortic stenosis is almost halved when a mechanical valve is implanted. ${ }^{26}$ Similarly, bioprosthetic AVR in young and middle-aged adults has been associated with a comparable survival deficit compared with age-matched general population. ${ }^{3}$ We postulate that the Ross procedure provides an opportunity to improve long-term outcomes. Registries and small, single-centred trials comparing the Ross procedure to conventional AVR suggest the Ross procedure in nonelderly patients requiring AVR in superior to conventional AVR. However, many of these trials were of low quality and uncertainty remains surrounding the actual risks and benefits of the Ross procedure. ${ }^{1415}$

It is now time to proceed with a multi-centre, expertisebased RCT powered to assess long-term clinically relevant outcomes comparing the Ross procedure with conventional AVR. We designed the REVIVAL trial to achieve this goal. Our network of cardiac surgery centres established in other large international trials (Steroids In caRdiac Surgery, ${ }^{27}$ Left Atrial Appendage Occlusion Study III, ${ }^{28}$ Coronary Artery Bypass Surgery Off or On Pump Revascularisation Study $\left.(\text { CORONARY })^{22}\right)$, has a sufficient number of Ross expert centres to undertake a definitive trial.

We have carefully examined the risks of the Ross procedure in two systematic reviews. ${ }^{11}{ }^{17}$ Given the totality of the data, we believe that the study we propose is safe and will yield a definitive answer with respect to the best care path for non-elderly adults requiring aortic valve surgery. The comparative review highlights similar perioperative risk with more favourable longer-term outcomes for Ross versus conventional AVR. The data are of low quality and likely biased given the studies are predominantly observational; however, the results are encouraging. The use of expertisebased randomisation recognises that not all surgeons should be performing the more complex Ross procedure and maintains safety for the study participants. Only a trial such as REVIVAL will establish whether the Ross pathway is safe and preferred. As described, a DSMB will closely monitor the trial for safety.

The pilot trial is limited by its sample size and will not be able to provide data on differences in outcomes between the Ross and conventional valve procedures. The trial is also limited in that after randomisation, members of the operative and perioperative care teams will not be blinded due to obvious logistical constraints. We cannot feasibly blind patients given potential surgeon changes following randomisation and due to requirement for oral anticoagulation with mechanical valves. Event adjudicators cannot be blinded, as event investigation will invariably lead to some amount of unblinding during review of hospital records. To account for the absence of blinding, we have chosen objective outcome definitions minimising the opportunity for bias in outcome assessment.

We will disseminate the final results at major meetings, journals, regional seminars and hospital rounds. Participating international key opinion leaders will present results at various regional and national forums ensuring a widespread audience. We will also disseminate the results of REVIVAL via the Reducing Global Perioperative Risk Multimedia Resource Centre (RGPR). RGPR is funded by the Ontario CIHR SPOR Support Unit, and hosted jointly by The Canadian Journal of Cardiology, The American Journal of Cardiology and The American Journal of Medicine. Designed according to the Canadian Knowledge-to-Action Framework, RGPR is a multimedia resource centre, housing knowledge product tools of our team's research, including slide and audio instructional videos, clinical practice guidelines, fact sheets, as well as full-text articles, links to abstracts and data summaries. To facilitate widespread knowledge 'push', RGPR is linked to Elsevier's entire online global readership. Opt-in email blasts and electronic banner advertisements (across subspecialty journals) allow for active exposure to over one million clinicians across the globe.

The results from the large-scale trial will be used in three ways. First, a positive trial will stimulate the training of more Ross experts globally, increasing its uptake. Second, the increased uptake will lead to a substantial reduction in morbidity and mortality in this young patient group. Third, the successful completion of REVIVAL trial will set a new bar for surgical valves trials. Currently, non-catheter based aortic valve trials have only focused on short-term and surrogate outcomes such as left ventricular mass regression. ${ }^{29}$

\section{CONCLUSION}

Due to the poor long-term outcomes among non-elderly patients requiring AVR, finding alternative approaches that minimise valve-related morbidity and mortality is imperative. REVIVAL will determine if the Ross procedure, in non-elderly adults, provides greater benefit than conventional valve prostheses. 
Author affiliations

${ }^{1}$ Surgery, McMaster University Faculty of Health Sciences, Hamilton, Ontario, Canada

${ }^{2}$ Medicine, McMaster University Faculty of Health Sciences, Hamilton, Ontario, Canada

${ }^{3}$ Department of Cardiac Surgery, KU Leuven University Hospitals Leuven, Leuven, Belgium

${ }^{4}$ Cardiac Surgery, University of Western Ontario Faculty of Health Sciences, London, Ontario, Canada

${ }^{5}$ McMaster University, Hamilton, Ontario, Canada

${ }^{6}$ Population Health Research Institute, Hamilton, Ontario, Canada

${ }^{7}$ FSBI National Medical Research Center named after E N Meshalkin, Novosibirsk, Novosibirskaâ, Russian Federation

${ }^{8}$ Cardiac Surgery, Bristol Royal Hospital for Children, Bristol, UK

Contributors All authors have contributed significantly to the content and meet the ICMJE criteria, and they have all read and approved of the final submission. RW, EB-C, MC and SS contributed significantly to the study's concept and design. RW and $\mathrm{HH}$ wrote the first draft of the manuscript, provided critical revisions, and gave final approval of the submitted manuscript. EB-C, MC, SS, FR, GRM, HH, PJD, SB, $\mathrm{JE}, \mathrm{KB}, \mathrm{RS}$ and $\mathrm{AB}-\mathrm{P}, \mathrm{SS}$ and PV provided critical revisions and gave final approval of the submitted manuscript.

Funding The REVIVAL pilot trial is funded by the Canadian Institutes of Health Research and McMaster University. RW is supported by a Heart and Stroke of Canada career award and holds a Canada Research Chair.

Competing interests RW reports grants from Bayer, Roche and BoeringerIngelheim outside the submitted work. He also reports speaker honorarium from Boeringer-Ingelheim outside the submitted work and consultancy for AtriCure and PhaseBio outside the submitted work. EB-C reports grants from Bayer and Roche outside the submitted work. MC reports speaker honoraria from Medtronic, Edwards Lifesciences, Terumo Aortic, Boston Scientific, and Abbott Vascular. JE reports consulting/honoraria support fom Astra-Zeneca, Bayer, BoehringerIngelheim, Bristol-Myer-Squibb, Daiichi-Sankyo, Eli-Lilly, Glaxo-Smith-Kline, Pfizer, Janssen, Sanofi-Aventis and Servier. He reports grants and/or in-kind support from Astra-Zeneca, Bayer, Boehringer-Ingelheim, Bristol-Myer-Squibb, Glaxo-Smith-Kline, Pfizer, Janssen, Sanofi-Aventis. PJD reports grants from Abbott Diagnostics, AstraZeneca, Bayer, Boehringer Ingelheim, Bristol-Myers-Squibb, Coviden, Octapharma, Philips Healthcare, Roche Diagnostics, Siemens and Stryker. PJD has participated in advisory board meetings for GlaxoSmithKline, Boehringer Ingelheim and Bayer. He also attended an expert panel meeting with AstraZeneca and Boehringer Ingelheim. FR reports a grant from Medtronic, and consultancy fees from Atricure \& LivaNova.

Patient and public involvement Patients and/or the public were involved in the design, or conduct, or reporting, or dissemination plans of this research. Refer to the Methods section for further details.

Patient consent for publication Not required.

Provenance and peer review Not commissioned; externally peer reviewed.

Open access This is an open access article distributed in accordance with the Creative Commons Attribution Non Commercial (CC BY-NC 4.0) license, which permits others to distribute, remix, adapt, build upon this work non-commercially, and license their derivative works on different terms, provided the original work is properly cited, appropriate credit is given, any changes made indicated, and the use is non-commercial. See: http://creativecommons.org/licenses/by-nc/4.0/.

ORCID iD

Richard Whitlock http://orcid.org/0000-0002-6863-5884

\section{REFERENCES}

1 Mazine A, Ouzounian M. Aortic valve replacement in young and middle-aged adults: looking beyond the tree that hides the forest. Ann Transl Med 2017;5:92.

2 Bouhout I, Stevens L-M, Mazine A, et al. Long-Term outcomes after elective isolated mechanical aortic valve replacement in young adults. J Thorac Cardiovasc Surg 2014;148:1341-6.
3 Bourguignon T, Bouquiaux-Stablo A-L, Candolfi P, et al. Very longterm outcomes of the Carpentier-Edwards Perimount valve in aortic position. Ann Thorac Surg 2015;99:831-7.

4 Puvimanasinghe JP, Steyerberg EW, Takkenberg JJ. Prognosis after aortic valve replacement with a bioprosthesis: predictions based on meta-analysis and microsimulation. Circulation 2001;103:1535-41.

5 Concha M, Aranda PJ, Casares J, et al. The Ross procedure. J Card Surg 2004;19:401-9.

6 Stelzer P. The Ross procedure: state of the art 2011. Semin Thorac Cardiovasc Surg 2011;23:115-23.

7 Mazine A, David TE, Rao V, et al. Long-Term outcomes of the Ross procedure versus mechanical aortic valve replacement. Circulation 2016;134:576-85.

8 David TE, David C, Woo A, et al. The Ross procedure: outcomes at 20 years. J Thorac Cardiovasc Surg 2014;147:85-94.

9 Schmidtke C, Stierle U, Sievers H-H, et al. [The Ross procedure (pulmonary autograft) as an alternative for aortic valve replacement]. Dtsch Med Wochenschr 2003;128:1759-64.

10 Mirkhani SH, Salehi M. Mid-Term results of the Ross operation. Arch Iran Med 2004;7:104-7.

11 McClure GR, Belley-Cote EP, Um K, et al. The Ross procedure versus prosthetic and homograft aortic valve replacement: a systematic review and meta-analysis. Eur J Cardio-Thoracic Surg 2019;55:247-55.

12 Stulak JM, Burkhart HM, Sundt TM, et al. Spectrum and outcome of reoperations after the Ross procedure. Circulation 2010;122:1153-8.

13 Rahimtoola SH. Choice of prosthetic heart valve in adults. J Am Coll Cardiol 2010;55:2413-26.

14 Klieverik LMA, Takkenberg JJM, Bekkers JA, et al. The Ross operation: a Trojan horse? Eur Heart J 2007;28:1993-2000.

15 Reece TB, Welke KF, O'Brien S, et al. Rethinking the Ross procedure in adults. Ann Thorac Surg 2014;97:175-81.

16 Sharabiani MTA, Dorobantu DM, Mahani AS, et al. Aortic Valve Replacement and the Ross Operation in Children and Young Adults. $J$ Am Coll Cardiol 2016;67:2858-70.

17 Sibilio S, Koziarz A, Belley-Côté EP, et al. Outcomes after Ross procedure in adult patients: a meta-analysis and microsimulation. $J$ Card Surg 2019;34:285-92.

18 Guyatt GH, Oxman AD, Schünemann HJ, et al. GRADE guidelines: a new series of articles in the Journal of clinical epidemiology. $J$ Clin Epidemiol 2011;64:380-2.

19 Devereaux PJ, Bhandari M, Clarke M, et al. Need for expertise based randomised controlled trials. BMJ 2005;330:88.

20 van der Linden W. On the generalization of surgical trial results. Acta Chir Scand 1980;146:229-34.

21 van der Linden W. Pitfalls in randomized surgical trials. Surgery 1980;87:258-62.

22 Lamy A, Devereaux PJ, Prabhakaran D, et al. Rationale and design of the coronary artery bypass grafting surgery off or on pump revascularization study: a large international randomized trial in cardiac surgery. Am Heart $J$ 2012;163:1-6.

23 Akl EA, Briel M, You JJ, et al. Potential impact on estimated treatment effects of information lost to follow-up in randomised controlled trials (LOST-IT): systematic review. BMJ 2012;344:e2809.

24 Lakatos E. Sample sizes based on the log-rank statistic in complex clinical trials. Biometrics 1988;44:229-41.

25 American Heart Association. Types of replacement heart valves; 2016.

26 Korteland NM, Etnel JRG, Arabkhani B, et al. Mechanical aortic valve replacement in non-elderly adults: meta-analysis and microsimulation. Eur Heart J 2017;38:3370-7.

27 Whitlock RP, Devereaux PJ, Teoh KH, et al. Methylprednisolone in patients undergoing cardiopulmonary bypass (SIRS): a randomised, double-blind, placebo-controlled trial. The Lancet 2015;386:1243-53.

28 Whitlock R, Healey J, Vincent J. Rationale and design of the left atrial appendage occlusion study (LAAOS) III. Ann. Cardiothorac. Surg 2014;3:45-54.

29 Suri RM, Zehr KJ, Sundt TM, et al. Left ventricular mass regression after porcine versus bovine aortic valve replacement: a randomized comparison. Ann Thorac Surg 2009;88:1232-7.

30 Goldstone AB, Chiu P, Baiocchi M, et al. Mechanical or biologic prostheses for aortic-valve and mitral-valve replacement. $N$ Engl J Med 2017;377:1847-57.

31 Etnel JRG, Huygens SA, Grashuis P, et al. Bioprosthetic aortic valve replacement in Nonelderly adults: a systematic review, meta-analysis, Microsimulation. Circ Cardiovasc Qual Outcomes 2019;12:e005481. 